\title{
Effects of folic acid fortification on orofacial clefts prevalence: a meta-analysis
}

\author{
Natalia Millacura ${ }^{1}$, Rosa Pardo ${ }^{2,3,4}$, Lucia Cifuentes ${ }^{5}$ and José Suazo ${ }^{1, *}$ \\ 'Instituto de Investigación en Ciencias Odontológicas, Facultad de Odontología, Universidad de Chile, Sergio \\ Livingstone \#943, Santiago, Chile: ${ }^{2}$ Sección de Genética, Hospital Clínico Universidad de Chile, Santiago, Chile: \\ ${ }^{3}$ Unidad de Neonatología, Hospital Clínico Universidad de Chile, Santiago, Chile: ${ }^{4}$ Unidad de Genética, Hospital \\ Dr Sótero del Río, Santiago, Chile: ${ }^{5}$ Programa de Genética Humana, Instituto de Ciencias Biomédicas, Facultad de \\ Medicina, Universidad de Chile, Santiago, Chile
}

Submitted 15 September 2016: Final revision received 31 March 2017: Accepted 10 April 2017: First published online 23 May 2017

\begin{abstract}
Objective: Orofacial clefts (OFC) are the most prevalent craniofacial birth defect. Folic acid (FA) supplementation has been demonstrated as an effective intervention to reduce risk of OFC occurrence. However, the effect of mandatory FA fortification of wheat and/or maize flour on OFC prevalence has shown controversial results among countries adopting this policy. Thus, we performed a meta-analysis to synthesize the available evidence evaluating the global impact of this mandatory policy on OFC occurrence.

Design: Literature search in conventional and grey medical/scientific databases showed fifteen studies considering OFC prevalence in pre- and post-fortification periods with FA. The effect of this policy was evaluated by computing relative risk (RR) and separating samples into total OFC, non-syndromic forms, cleft lip with or without cleft palate $(\mathrm{CL} / \mathrm{P})$ and cleft palate only $(\mathrm{CPO})$.

Results: We found a significant effect of FA fortification only on non-syndromic $\mathrm{CL} / \mathrm{P}(\mathrm{RR}=0 \cdot 88 ; 95 \% \mathrm{CI} 0 \cdot 81,0 \cdot 96)$, whereas neutral effects were detected for total OFC (syndromic plus non-syndromic) and CPO.

Conclusions: Our results may reflect the different aetiology of syndromic OFC with respect to non-syndromic forms and the $\mathrm{CL} / \mathrm{P}$ related to $\mathrm{CPO}$. Although the number of non-syndromic CL/P samples was lower than that for total OFC, the absence of both between-study heterogeneity and publication bias leads us to conclude that FA fortification may have beneficial effects on non-syndromic CL/P.
\end{abstract}

\author{
Keywords \\ Orofacial clefts \\ Folic acid fortification \\ Meta-analysis
}

Orofacial clefts (OFC) are among the most common birth defects worldwide, constituting the main disorders affecting craniofacial structures ${ }^{(1)}$. Their prevalence shows variation according to factors such as ethnic origin and socio-economic status ${ }^{(2,3)}$. OFC have been classified as cleft palate only (CPO), cleft lip only (CL) and cleft lip with cleft palate (CLP). These latter two categories are grouped as cleft lip with or without cleft palate $(\mathrm{CL} / \mathrm{P})^{(4)}$. About $70 \%$ of OFC occur as a non-syndromic condition (NSOFC) without any other apparent structural or cognitive abnormality, while the remaining $30 \%$ are found as part of more than 300 recognizable genetic syndromes ${ }^{(3,4)}$. OFC constitute a worldwide public health problem due to their prevalence, complex rehabilitation plus medical costs, and emotional burden to patients and their families. In this context, OFC patients present a wide variety of medical complications in early processes such as feeding, speaking and hearing and in their social integration ${ }^{(5)}$. In addition, these patients have a higher risk of certain cancers and psychiatric disorders in adult life ${ }^{(6)}$.

The aetiology of OFC can be explained by the interaction between functionally altered genes plus environmental factors $^{(5)}$. Probably the best example of a gene-environmental interaction in clefts' aetiology is folate/folic acid (FA) metabolism $^{(7)}$. Folates are involved in the transfer of methyl groups (one-carbon units) to DNA, being an epigenetic mechanism of gene expression modulation ${ }^{(8,9)}$. Maternal folate/methyl-donor status appears to play a central role during early embryonic development where its deficit affects embryo and fetal cells with high proliferation rates such as 
neural crest cells ${ }^{(7)}$. These cells notably contribute to maxillofacial bone and cartilage development by means of an epigenetic-regulated differentiation ${ }^{(9)}$. Subsequently, epigenetic mechanisms modulate the secondary palate development as has been demonstrated in animal mod$\mathrm{els}^{(10)}$. Compared with mothers of non-affected children, mothers of cleft cases had a diet poor in folates ${ }^{(11)}$. In this context, multi-ethnic systematic reviews have demonstrated that maternal periconceptional FA supplementation is an effective intervention preventing $\mathrm{OFC}^{(12-14)}$ while other authors showed a neutral pooled effect ${ }^{(15)}$. Thus, the FAOFC relationship is still an issue that needs further studies.

In January 2000, the Chilean Ministry of Health established a mandatory fortification of wheat flour with $\mathrm{FA}^{(16)}$. This national public health policy led to a significant reduction (about 50\%) in the prevalence of neural tube defects $^{(17,18)}$. Despite the above-mentioned role of FA supplementation in preventing OFC, in several countries the effect of FA fortification on its prevalence is controversial. Some studies in the Chilean population did not show benefits on OFC reduction ${ }^{(18,19)}$; however, other authors registered no effects for $\mathrm{CL} / \mathrm{P}$ but a significant increase in CPO prevalence in the country ${ }^{(20,21)}$. On the other hand, in Iran a significant decrease of NSOFC was observed as a response to the mandatory FA fortification $^{(22)}$. Therefore we aimed to perform a meta-analysis based on published studies comparing prevalence rates for all OFC types in FA pre-fortification $v$. post-fortification periods in order to contribute to solving this controversy.

\section{Methods}

\section{Literature search and quality assessment of single studies}

In order to minimize the risk of bias, we performed a search based on the following considerations. (i) A search was conducted in diverse conventional scientific literature databases: Cochrane Library, EMBASE, PubMed, ScienceDirect, Scielo, Springerlink and Web of Science; and in grey literature databases: GreyNet, GreyLit, LILACS, Open Grey and POPLINE (Fig. 1). The literature search was conducted through 15 August 2016 with no date restrictions for early studies, considered the terms 'cleft lip palate' OR 'cleft palate' OR 'orofacial clefts' AND 'folic acid', and was restricted to English and Spanish languages. (ii) The search was performed independently by two authors (N.M. and J.S.) who extracted the following data from each report: authors, year of publication, country of origin of the samples, number of OFC cases and total births for pre-fortification period, and number of OFC cases and total births for post-fortification period. (iii) For samples from the same country/region/state, we confirmed that different time lapses for pre- and/or postfortification periods were considered. (iv) We performed a quality assessment for each study identified using the
Newcastle-Ottawa Scale (NOS), considering the selection criteria of pre- and post-fortification samples, comparability of these groups, and the ascertainment of either the exposure or outcome. NOS assigns a maximum score of 9 points where studies showing $<5$ points have high risk of bias and limitations, with these being excluded from a meta-analysis $^{(23)}$.

\section{Statistical methods}

Meta-analysis was performed comparing OFC prevalence between pre- and post-fortification periods considering all births in a region, state or hospitals within a country. The effect was estimated by means of the relative risk (RR) with $95 \%$ CI for each study and for the pooled effect. The presence of between-study heterogeneity was evaluated by the Cochran $Q$ statistic, which is the base for the $I^{2}$ test expressing the percentage of between-study variability explained by heterogeneity ${ }^{(24)}$. The pooled effect was estimated using fixed-effects or random-effects methods based on the absence $\left(I^{2}<50\right)$ or presence of heterogeneity $\left(I^{2}>50\right)$, respectively ${ }^{(25)}$. In the presence of heterogeneity and in order to identify its sources, we additionally applied a univariate meta-regression ${ }^{(25)}$ between each study effect and three covariates: total sample size, the time lapse (months) between the pre- and post-fortification, and the FA mean daily dose reached by fortification in each country based on the Food Fortification Initiative ${ }^{(26)}$. Publication bias (i.e. studies which have been published based on their sample size and their positive or negative effect) was evaluated using Begg's funnel plot ${ }^{(25)}$. If a meta-analysis includes studies of mainly small sample size and/or a low number of studies $(\leq 10)$, the funnel-based method loses power ${ }^{(27)}$. Therefore, in this case, publication bias was alternatively assessed via cumulative meta-analysis by precision ${ }^{(27)}$. A sensitivity analysis was performed to assess the robustness of meta-analysis based on the detection of significant changes related to the overall effect when one study is dropped at a time and the pooled effect is recalculated (leave-one-out method) ${ }^{(25)}$. All tests were performed using the statistical OpenMeta (Analyst) package ${ }^{(28)}$.

\section{Results}

Figure 1 summarizes the steps and results of our search in order to find a group of publications complying with the criteria to achieve the aim of our study and with proper quality. The initial search showed a total of 961 reports. After discarding duplicated studies, we evaluated 653 reported abstracts. Then, 356 articles met the criteria to be analysed at full text level. Finally, fifteen studies were considered in the quality analysis (NOS) and all of them were included for pooled effects of FA fortification on OFC risk. Data extracted from the selected articles are detailed in Table $1^{(18-22,29-38)}$. If a study included samples from two or more countries, these samples were evaluated separately. 


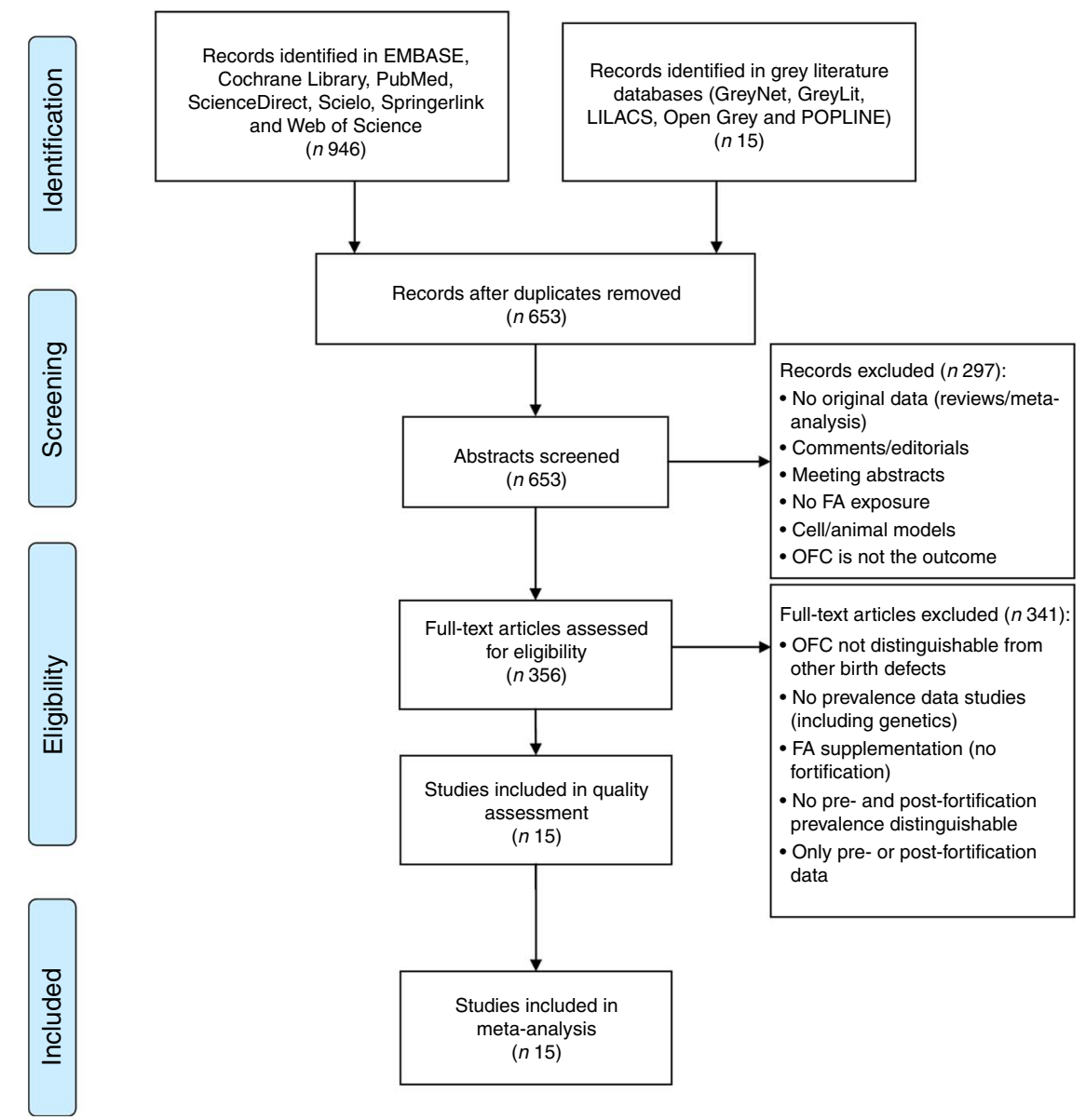

Fig. 1 PRISMA (Preferred Reporting Items for Systematic Reviews and Meta-Analyses) flowchart showing selection of studies for the present meta-analysis on the effects of folic acid (FA) fortification on orofacial clefts (OFC) prevalence

The first analysis included all OFC considering the fifteen studies selected where syndromic and nonsyndromic cases were grouped. These reports comprised twenty-four samples from different countries or regions in a same country (Table 1). We detected between-study heterogeneity ( $I^{2}=78.5 \%$; Fig. 2$)$. Thus, a random-effects model meta-analysis was applied, which showed a non-significant influence of FA fortification on OFC prevalence $(\mathrm{RR}=0.97 ; 95 \% \mathrm{CI} 0.92,1.02$; Fig. 2). Univariate meta-regression recognized the additional FA daily dose reached by fortification as a source of heterogeneity (regression coefficient $=-0.576 ; 95 \%$ CI $-1 \cdot 022,-0 \cdot 130$; see online supplementary material, Table S1). The funnel plot exhibited asymmetry (see online supplementary material, Fig. S1(a)), demonstrating the presence of publication bias. Sensitivity analysis supported the robustness of the meta-analysis results (data not shown). Then, we identified the studies where $\mathrm{CL} / \mathrm{P}$ and $\mathrm{CPO}$ data were analysed separately (ten articles including sixteen samples). For $\mathrm{CL} / \mathrm{P}$ we found evidence of heterogeneity $\left(I^{2}=79.5 \%\right.$; Fig. 3) and the pooled effect based on a random-effects model showed no significant effect of fortification ( $R R=$ 0.99; 95\% CI 0.92, 1.06; Fig. 3). Univariate meta-regression did not detect heterogeneity sources (Table S1). Cumulative meta-analysis by precision demonstrated the absence of publication bias (Fig. S1(b)). However, the sensitivity analysis showed that our results lacked robustness, where the exclusion of samples from Texas ${ }^{(32)}$ and Brazil ${ }^{(18)}$ respectively significantly decreased $(\mathrm{RR}=0 \cdot 73 ; 95 \% \mathrm{CI} 0 \cdot 67,0 \cdot 80)$ and increased $\mathrm{CL} / \mathrm{P}$ risk $(\mathrm{RR}=1 \cdot 32 ; 95 \% \mathrm{CI} 1 \cdot 09$, 1.60; data not shown). Fortification also showed a non-significant effect on CPO prevalence based on a random-effects model $\left(I^{2}=90 \cdot 2 \% ; \mathrm{RR}=1 \cdot 02 ; 95 \%\right.$ CI $0.89,1 \cdot 18 ;$ Fig. 4). This heterogeneity could not be explained by any of the analysed covariates (Table S1). Sensitivity analysis demonstrated the robustness of our results (data not shown) but cumulative meta-analysis by precision showed evidence of publication bias (Fig. S1(c)).

Only in five studies (comprising seven samples) was it possible to identify non-syndromic cases from syndromic cases of OFC (Table 1). Random-effects meta-analysis showed that FA fortification had no effect on NSOFC prevalence $\left(I^{2}=54.6 \%\right.$; RR $=0.92 ; 95 \%$ CI $0.83,1.03$; Fig. 5). This heterogeneity may be explained by the total sample size of each study, which was negatively associated with the effect (regression coefficient $=-0 \cdot 004$; 
Table 1 Description of the studies and samples in the present meta-analysis on the effects of folic acid (FA) fortification on orofacial clefts prevalence

\begin{tabular}{|c|c|c|c|c|c|c|c|c|c|c|}
\hline Study, year, reference & Country (region) & $\begin{array}{l}\text { Pre-F } \\
\text { period }\end{array}$ & $\begin{array}{l}\text { Post-F } \\
\text { period }\end{array}$ & $\begin{array}{l}\text { No. of cases } \\
\text { Pre-F }\end{array}$ & $\begin{array}{l}\text { Total births } \\
\text { Pre-F }\end{array}$ & $\begin{array}{l}\text { No. of cases } \\
\text { Post-F }\end{array}$ & $\begin{array}{l}\text { Total births } \\
\text { Post-F }\end{array}$ & $\mathrm{NS}(\mathrm{S})$ & $\mathrm{CL} / \mathrm{P}(\mathrm{CPO})$ & NOS score \\
\hline Ray et al. (2003) ${ }^{(29)}$ & Canada (Ontario) & 1994-1997 & $1998-2000$ & 251 & 218977 & 143 & 117992 & $(+)$ & $(-)$ & 7 \\
\hline \multirow{4}{*}{ Castilla et al. $(2003)^{(19)}$} & Argentina & 1999 & $2000-2001$ & 91 & 51123 & 144 & 102747 & $(-)$ & $(-)$ & 7 \\
\hline & Brazil & 1999 & $2000-2001$ & 45 & 27767 & 69 & 55413 & $(-)$ & $(-)$ & 7 \\
\hline & Chile & 1999 & $2000-2001$ & 15 & 10755 & 29 & 24439 & $(-)$ & $(-)$ & 7 \\
\hline & Uruguay & 1999 & $2000-2001$ & 10 & 11145 & 19 & 21707 & $(-)$ & $(-)$ & 7 \\
\hline Simmons et al. $(2004)^{(30)}$ & USA (Arkansas) & 1993-1995 & $1999-2000$ & 204 & 104082 & 126 & 74556 & $(-)$ & $(+)$ & 8 \\
\hline Canfield et al. (2005) & USA & $1995-1996$ & $1999-2000$ & 4465 & 2881094 & 4227 & 2950761 & $(-)$ & $(+)$ & 8 \\
\hline Hashmi et al. (2005) (32) & USA (Texas) & $1995-1997$ & 1998-1999 & 481 & 541231 & 320 & 373002 & $(+)$ & $(+)$ & 8 \\
\hline \multirow[t]{5}{*}{ Botto et al. $(2006)^{(33)}$} & USA (Texas) & $1996-1998$ & $1999-2003$ & 1007 & 595858 & 2884 & 1838113 & $(-)$ & $(+)$ & 7 \\
\hline & USA (Atlanta) & $1985-1998$ & $1999-2003$ & 1267 & 820065 & 341 & 250551 & $(-)$ & $(+)$ & 7 \\
\hline & Australia (Victoria) & $1983-1995$ & $1996-2003$ & 1481 & 825069 & 944 & 501327 & $(-)$ & $(+)$ & 7 \\
\hline & Australia (Western) & $1980-1995$ & $1996-2003$ & 827 & 386088 & 498 & 201946 & $(-)$ & $(+)$ & 7 \\
\hline & Canada (Alberta) & 1997-1998 & 1999-2003 & 156 & 74605 & 386 & 190995 & $(-)$ & $(+)$ & 7 \\
\hline Yazdy et al. (2007) & USA & $1990-1996$ & 1998-2002 & 21057 & 24726932 & 12139 & 15143273 & $(-)$ & $(-)$ & 8 \\
\hline Nazer et al. (2007) & Chile & $1982-1999$ & $2001-2003$ & 223 & 140045 & 62 & 34370 & $(-)^{\prime}$ & $(+)$ & 7 \\
\hline Godwin et al. (2008) & Canada (Alberta) & $1992-1996$ & $1999-2003$ & 385 & 198321 & 374 & 191028 & $(-)$ & $(+)$ & 7 \\
\hline Sayed et al. (2008) ${ }^{(36)}$ & South Africa & $2001-2003$ & $2005-2006$ & 33 & 79587 & 18 & 46021 & $(-)$ & $(-)$ & 7 \\
\hline \multirow{3}{*}{ López-Camelo et al. (2010) ${ }^{(18)}$} & Argentina & $1982-2001$ & $2005-2007$ & 1965 & 1302290 & 175 & 147853 & $(+)$ & $(+)$ & 7 \\
\hline & Brazil & 1982-2002 & $2005-2007$ & 1254 & 911920 & 160 & 92843 & $(+)$ & $(+)$ & 7 \\
\hline & Chile & $1982-1997$ & $2002-2007$ & 432 & 283403 & 436 & 243624 & $(+)$ & $(+)$ & 7 \\
\hline Souza and Raskin $(2013)^{(37)}$ & Brazil (Parana) & 2002-2004 & $2006-2008$ & 513 & 482094 & 418 & 452589 & $(+)$ & $(+)$ & 8 \\
\hline Nazer and Cifuentes (2014) & Chile & $1982-1994$ & $2001-2010$ & 391 & 241021 & 570 & 282568 & $(-)$ & $(+)$ & 7 \\
\hline Golalipour et al. (2014) (22) & Iran (Northern) & $2006-2007$ & $2008-2009$ & 22 & 34295 & 15 & 46249 & $(+)$ & $(-)$ & 7 \\
\hline Yang et al. $(2016)^{(38)}$ & USA (California) & $1989-1996$ & $1999-2000$ & 946 & 477716 & 1337 & 776983 & $(-)$ & $(+)$ & 7 \\
\hline Total & & & & 37521 & 35425483 & 25834 & 24160950 & & & \\
\hline
\end{tabular}

Pre-F, FA pre-fortification period; Post-F, FA post-fortification period; NS(S), studies where non-syndromic and syndromic clefts are recognizable; CL/P(CPO), studies where cleft lip with or without cleft palate (CL/P) and cleft lip palate only (CPO) are recognizable; NOS score, score from Newcastle-Ottawa Scale ${ }^{(23)}$. 


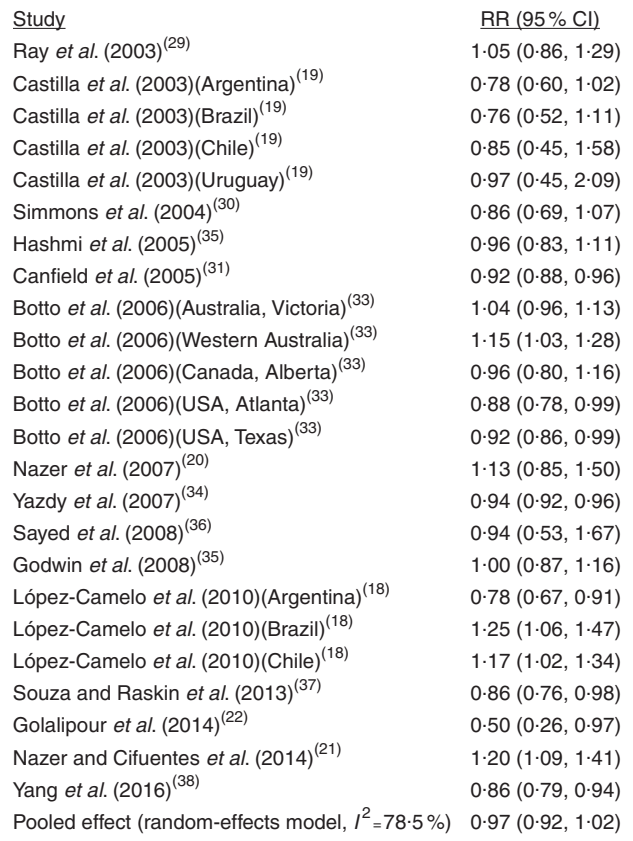

\begin{tabular}{cc}
$\underline{R R(95 \% \mathrm{Cl})}$ & Weight $(\%)$ \\
\cline { 2 - 2 } $1.05(0.86,1.29)$ & 3.19 \\
$0.78(0.60,1.02)$ & 2.27 \\
$0.76(0.52,1.11)$ & 1.27 \\
$0.85(0.45,1.58)$ & 0.51 \\
$0.97(0.45,2.09)$ & 0.34 \\
$0.86(0.69,1.07)$ & 2.87 \\
$0.96(0.83,1.11)$ & 4.80 \\
$0.92(0.88,0.96)$ & 8.21 \\
$1.04(0.96,1.13)$ & 6.90 \\
$1.15(1.03,1.28)$ & 5.81 \\
$0.96(0.80,1.16)$ & 3.60 \\
$0.88(0.78,0.99)$ & 5.52 \\
$0.92(0.86,0.99)$ & 7.26 \\
$1.13(0.85,1.50)$ & 2.04 \\
$0.94(0.92,0.96)$ & 8.64 \\
$0.94(0.53,1.67)$ & 0.59 \\
$1.00(0.87,1.16)$ & 4.77 \\
$0.78(0.67,0.91)$ & 4.40 \\
$1.25(1.06,1.47)$ & 4.13 \\
$1.17(1.02,1.34)$ & 5.06 \\
$0.86(0.76,0.98)$ & 5.19 \\
$0.50(0.26,0.97)$ & 0.46 \\
$1.20(1.09,1.41)$ & 5.21 \\
$0.86(0.79,0.94)$ & 6.84 \\
$0.97(0.92,1.02)$ & \\
&
\end{tabular}

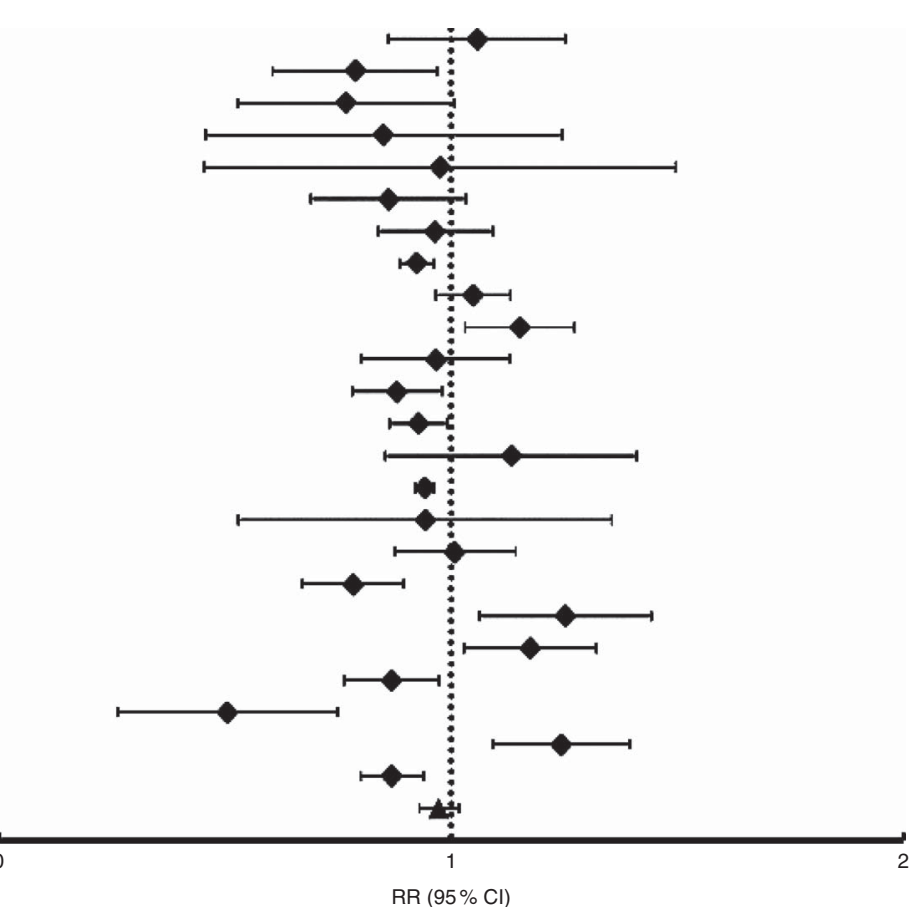

Fig. 2 Forest plot showing the effect of folic acid fortification on the prevalence of total orofacial clefts (total OFC). The study-specific relative risk $(\mathrm{RR})$ and $95 \% \mathrm{Cl}$ are represented by the black square and horizontal line, respectively; the black triangle represents the pooled RR and the horizontal line represents the pooled $95 \% \mathrm{Cl}$; the dotted line at $\mathrm{RR}=1$ represents the null effect

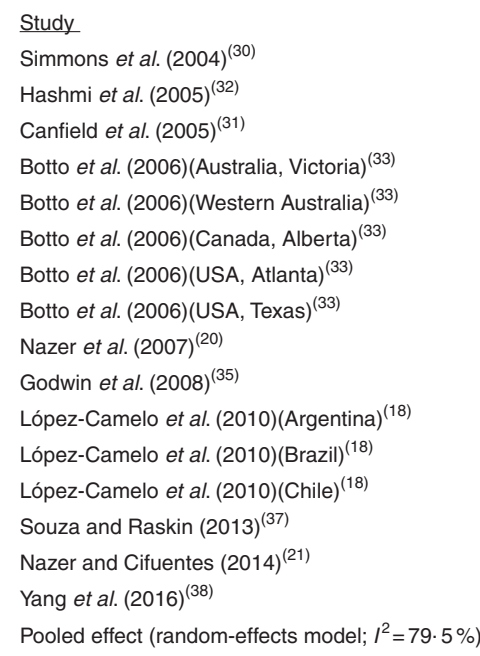

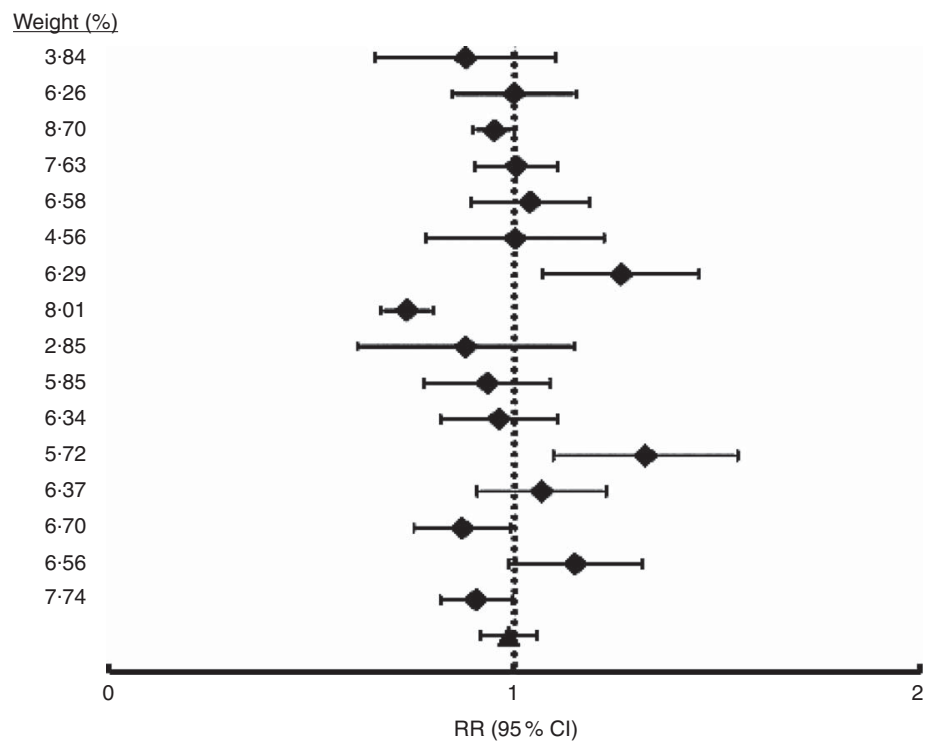

Fig. 3 Forest plot showing the effect of folic acid fortification on the prevalence of cleft lip with or without cleft palate (CL/P). The study-specific relative risk $(\mathrm{RR})$ and $95 \% \mathrm{Cl}$ are represented by the black square and horizontal line, respectively; the black triangle represents the pooled RR and the horizontal line represents the pooled $95 \% \mathrm{Cl}$; the dotted line at $\mathrm{RR}=1$ represents the null effect

95\% CI -0.003, -0.005; Table S1). Sensitivity analysis showed that upon exclusion of the report from Ontario ${ }^{(29)}$ the effect of FA fortification became significant $(\mathrm{RR}=0.90$; 95\% CI 0.82, 0.98; data not shown). There was no substantial evidence of publication bias from cumulative meta-analysis by precision (Fig. S1(d)). Only three studies (five samples) considered NSCL/P and NSCPO classification. Fixed-effects model meta-analysis for NSCL/P demonstrated a significant reduction of its risk after FA fortification $\left(I^{2}=22 \cdot 4 \%\right.$; RR $=0 \cdot 88 ; 95 \%$ CI 0.81, 0.96; Fig. 6). These results are supported by both the absence of publication bias according to the cumulative meta-analysis (Fig. S1(e)) and the robustness of our findings based on the sensitivity analysis (data not shown). On the contrary, NSCPO meta-analysis exhibited heterogeneity and no significant effect of FA fortification on its prevalence 
Study

Simmons et al. (2004) ${ }^{(30)}$

Hashmi et al. (2005) ${ }^{(32)}$

Canfield et al. (2005) $)^{(31)}$

Botto et al. (2006)(Australia, Victoria) ${ }^{(33)}$

Botto et al. (2006)(Western Australia) ${ }^{(33)}$

Botto et al. (2006)(Canada, Alberta) ${ }^{(33)}$

Botto et al. (2006)(USA, Atlanta) ${ }^{(33)}$

Botto et al. (2006)(USA, Texas) ${ }^{(33)}$

Nazer et al. (2007) ${ }^{(20)}$

Godwin et al. (2008) ${ }^{(35)}$

López-Camelo et al. (2010)(Argentina) ${ }^{(18)}$

López-Camelo et al. (2010)(Brazil) ${ }^{(18)}$

López-Camelo et al. (2010)(Chile) ${ }^{(18)}$

Souza and Raskin (2013) $)^{(37)}$

Nazer and Cifuentes (2014) ${ }^{(21)}$

Yang et al. (2016) ${ }^{(38)}$

Pooled effect (random-effects model; $\left.I^{2}=90 \cdot 2 \%\right) 1 \cdot 02(0 \cdot 89,1 \cdot 18)$
$\underline{\mathrm{RR}(95 \% \mathrm{Cl}) \quad \text { Weight (\%) }}$

$0.84(0.59,1.19) \quad 5.31$

$0.88(0.67,1.15) \quad 6.09$

$0.88(0.82,0.95) \quad 7.67$

$1.11(0.98,1.26) \quad 7.37$

$1.29(1.10,1.52) \quad 7.09$

$0.92(0.69,1.22) \quad 5.95$

$1.75(1.43,2 \cdot 14) \quad 6.75$

$0.77(0.68,0.87) \quad 7.36$

$1 \cdot 80(1 \cdot 15,2 \cdot 84) \quad 4.34$

$1.13(0.90,1.42) \quad 6.53$

$1.43(1.13,1.81) \quad 6.42$

$0.27(0.16,0.45) \quad 3.85$

$1.08(0.77,1.50) \quad 5.48$

$0.86(0.66,1.12) \quad 6.12$

$1.48(1.17,1.88) \quad 6.41$

$0.81(0.70,0.93) \quad 7.26$

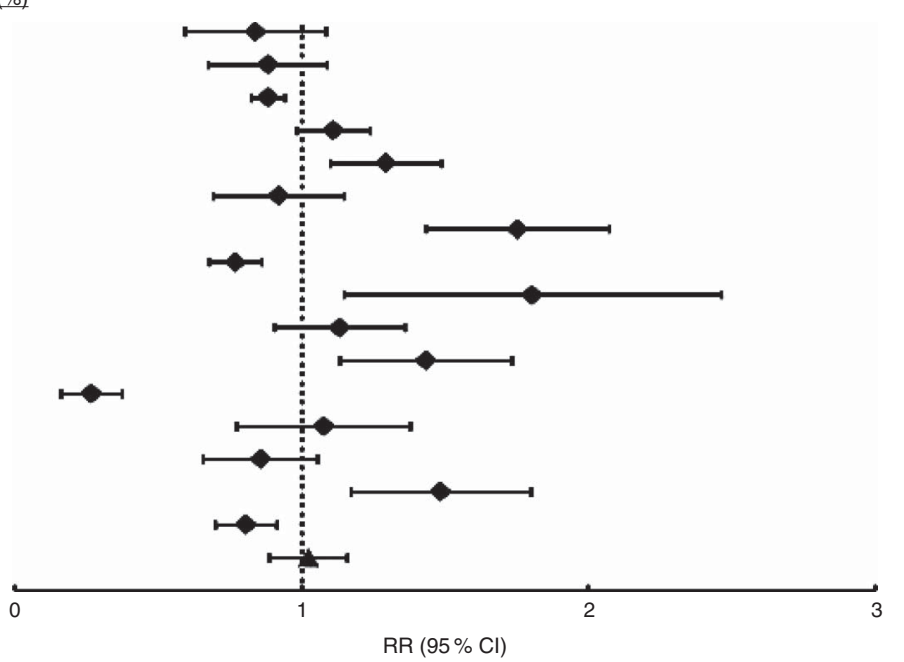

Fig. 4 Forest plot showing the effect of folic acid fortification on the prevalence of cleft palate only (CPO). The study-specific relative risk $(\mathrm{RR})$ and $95 \% \mathrm{Cl}$ are represented by the black square and horizontal line, respectively; the black triangle represents the pooled $\mathrm{RR}$ and the horizontal line represents the pooled $95 \% \mathrm{Cl}$; the dotted line at $\mathrm{RR}=1$ represents the null effect

Study

Ray et al. (2003) $)^{(29)}$

Hashmi et al. (2005) ${ }^{(32)}$

López-Camelo et al. (2010)(Argentina) ${ }^{(18)}$

López-Camelo et al. (2010)(Brazil) ${ }^{(18)}$

López-Camelo et al. (2010)(Chile) ${ }^{(18)}$

Souza and Raskin (2013) ${ }^{(37)}$

Golalipour et al. (2014) ${ }^{(22)}$

Pooled effect (random-effects model; $I^{2}=54.6 \%$ )

$\begin{array}{cc}\text { RR }(95 \% \mathrm{Cl}) & \text { Weight }(\%) \\ 1.19(0.95,1.50) & 12.47 \\ 0.97(0.84,1.11) & 19.58 \\ 0.80(0.66,0.96) & 15.78 \\ 0.89(0.70,1.14) & 11.52 \\ 1.01(0.85,1.19) & 17.43 \\ 0.87(0.76,0.99) & 20.74 \\ 0.50(0.26,0.97) & 2.48 \\ 0.92(0.83,1.03) & \end{array}$

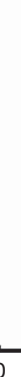

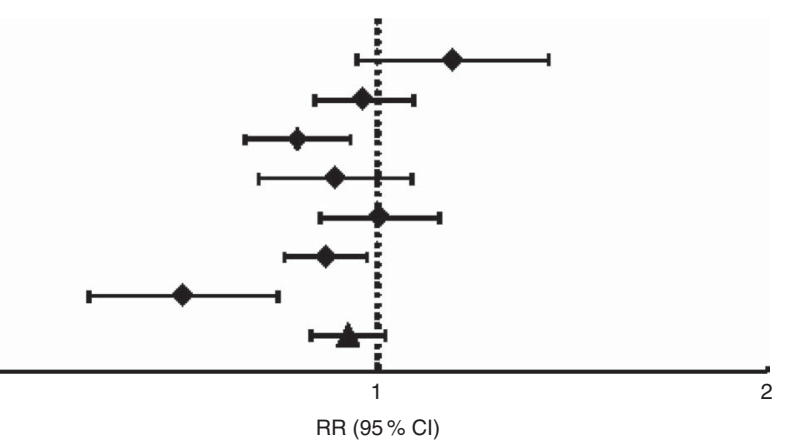

Fig. 5 Forest plot showing the effect of folic acid fortification on the prevalence of non-syndromic orofacial clefts (NSOFC). The study-specific relative risk (RR) and $95 \% \mathrm{Cl}$ are represented by the black square and horizontal line, respectively; the black triangle represents the pooled $\mathrm{RR}$ and the horizontal line represents the pooled $95 \% \mathrm{Cl}$; the dotted line at $\mathrm{RR}=1$ represents the null effect

\begin{tabular}{|c|c|c|}
\hline Study & $\underline{\mathrm{RR}}(95 \% \mathrm{Cl})$ & Weight (\%) \\
\hline Hashmi et al. (2005) $)^{(32)}$ & $1.00(0.85,1 \cdot 18)$ & $24 \cdot 65$ \\
\hline López-Camelo et al. (2010)(Argentina) ${ }^{(18)}$ & $0.75(0.61,0.92)$ & $16 \cdot 17$ \\
\hline López-Camelo et al. (2010)(Brazil) ${ }^{(18)}$ & $0.95(0.73,1 \cdot 24)$ & $9 \cdot 85$ \\
\hline López-Camelo et al. (2010)(Chile) ${ }^{(18)}$ & $0 \cdot 85(0 \cdot 70,1 \cdot 03)$ & $18 \cdot 15$ \\
\hline Souza and Raskin (2013) ${ }^{(37)}$ & $0.87(0.75,1.01)$ & $31 \cdot 18$ \\
\hline Pooled effect (fixed-effects model; $I^{2}=22 \cdot 4 \%$ ) & $0.88(0.81,0.96)$ & \\
\hline
\end{tabular}

Fig. 6 Forest plot showing the effect of folic acid fortification on the prevalence of non-syndromic cleft lip with or without cleft palate (NSCL/P). The study-specific relative risk (RR) and $95 \% \mathrm{Cl}$ are represented by the black square and horizontal line, respectively; the black triangle represents the pooled $\mathrm{RR}$ and the horizontal line represents the pooled $95 \% \mathrm{Cl}$; the dotted line at $\mathrm{RR}=1$ represents the null effect

$\left(I^{2}=71.1 \% ; \mathrm{RR}=1.00 ; 95 \%\right.$ CI $0.75,1.34 ;$ Fig. 7$)$. We did not find sources for this heterogeneity among the covariates considered for meta-regression (Table S1). Cumulative meta-analysis by precision showed no conclusive evidence of publication bias for NSCPO (Fig. S1(e)), while sensitivity analysis demonstrated the robustness of this last meta-analysis (data not shown).

\section{Discussion}

Our study presents certain characteristics which may be considered as strengths: a wide bibliographical search in several medical/scientific conventional and grey databases, where all of the included reports showed a high quality level (Table 1). Thus, these features allow us to consider that all 


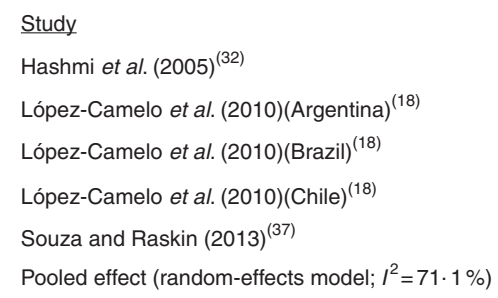

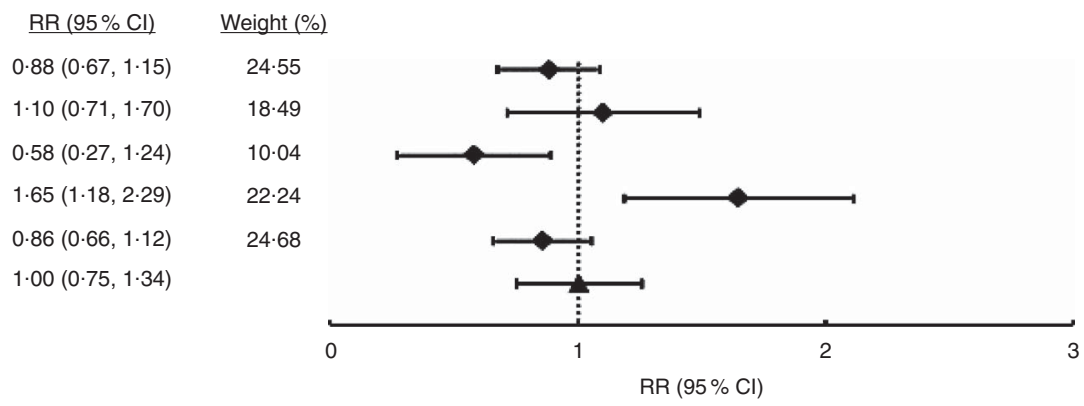

Fig. 7 Forest plot showing the effect of folic acid fortification on the prevalence of non-syndromic cleft palate only (NSCPO). The study-specific relative risk (RR) and $95 \% \mathrm{Cl}$ are represented by the black square and horizontal line, respectively; the black triangle represents the pooled RR and the horizontal line represents the pooled $95 \% \mathrm{Cl}$; the dotted line at $\mathrm{RR}=1$ represents the null effect

studies included in our meta-analysis have a low risk of bias. On the other hand, the great majority of our analyses exhibited between-study heterogeneity (with the exception of NSCL/P meta-analysis), results reflecting a limitation of the present report. However, we decided to search for sources of this heterogeneity instead of not considering these pooled effects. Univariate meta-regression discovered the possible heterogeneity sources in some cases (Table S1). For all OFC, FA dose was negatively associated with cleft risk, which is related to evidence showing a decrease in cleft occurrence by periconceptional FA use in a dose-dependent manner ${ }^{(39,40)}$. Additionally, for NSOFC we found that between-study heterogeneity may be explained by the sample size of each study. Sample size variation (directly associated to the precision of each study) is always an important factor affecting heterogeneity in meta-analysis ${ }^{(41)}$. The between-study variability detected could also be explained by variables not included in the meta-regression, such as the ethnic origin of the populations. Samples considered herein came from South and North America, Africa, Asia and Oceania. In this context, the response to FA consumption may be modulated by variants in folate/onecarbon metabolism genes ${ }^{(7)}$ such as the functional polymorphism $677 \mathrm{C} \rightarrow \mathrm{T}$ within the MTHFR gene coding a central enzyme (methylenetetrahydrofolate reductase) in this metabolism ${ }^{(42)}$. Its allelic frequency differs according to the ethnicity of each population (based on dbSNP information $)^{(43)}$. We decided to not perform meta-analysis segregating samples for continent in order to avoid a decrease in statistical power associated with forming subgroups. For total OFC and CPO, we found evidence of publication bias; this is another limitation. In this context, the report of Castilla et al. ${ }^{(19)}$, using samples from the Latin American Collaborative Study of Congenital Malformations (ECLAMC), excluded samples from certain countries where a low number of annual births $(<10000)$ was registered in the participating hospitals, contributing to this bias.

We applied the NOS in order to detect low-quality reports which have high risk of bias ${ }^{(23)}$. However, this scale was designed to be applied on case-control or cohort studies. As was commented by Simmons et al. ${ }^{(30)}$, all reports included here are considered ecological studies which may introduce an ecological bias possibly not detected by the NOS. A bias associated with this class of design is related to the latency time since exposure and effect measurement ${ }^{(44)}$. In the current report, some studies did not consider a time lapse between pre- and postfortification periods while other authors only included births registered months or years after the implementation of the mandatory FA policy (Table 1). Although this covariate was not detected as a source of heterogeneity when a meta-regression was applied (Table S1), it may introduce variability because the additional dose of FA does not homogeneously reach all people following application of the policy $^{(19)}$.

Regarding the effects of FA fortification policy, our meta-analysis found a beneficial effect only for NSCL/P prevalence (Fig. 6) and neutral influences on the other classifications of clefts. Although the NSCL/P analysis considered a reduced number of studies (three studies with five samples), we found support for the relative robustness of our results. Thus, we did not find evidence of betweenstudy heterogeneity (Fig. 6) or publication bias (Fig. S1(e)), and the combined effect remained consistent when any sample was dropped in the sensitivity analysis (data not shown). The different NSCL/P results in comparison with the other cleft categories may be explained by several factors. First, total OFC include both syndromic and nonsyndromic forms. The great majority of the syndromic forms have known causes associated with teratogens, single-locus mutations and chromosomal rearrangements ${ }^{(45)}$. Thus, the inclusion of syndromic clefts may lead our results to neutral effects of FA. However, FA fortification did not show a significant effect on NSOFC prevalence, possibly as a consequence of an admixture of NSCPO and NSCL/P, which have been considered different aetiological entities. Epidemiological data show a higher frequency of $\mathrm{CL} / \mathrm{P}$ in males than females, which is inverted for $\mathrm{CPO}^{(3)}$. In addition, $\mathrm{CPO}$ is more frequent in syndromes than $\mathrm{CL} / \mathrm{P}$ (50 and $30 \%$, respectively) ${ }^{(3)}$. These differences seem to reflect the genetic and embryological origins of these clefts, where $\mathrm{CL} / \mathrm{P}$ is associated with primary palate development whereas $\mathrm{CPO}$ is related to secondary palate closure, both considered independent events ${ }^{(3,46)}$. 
To the best of our knowledge, there is only one previous meta-analysis ${ }^{(47)}$ analysing the influence of FA fortification on OFC prevalence (grouping syndromic and non-syndromic forms). That meta-analysis considered only three studies which have also been included in the current report $^{(30,31,33)}$. The previous meta-analysis found a significant but marginal decrease in the risk of $\mathrm{CL} / \mathrm{P}$ after FA fortification which was not observed for CPO, supporting the hypothesis of a different aetiology among them. Those authors concluded that FA appears to play a minor or any role in OFC expression. Although we included a higher number of studies, our results lead us to the same conclusion as Johnson and Little ${ }^{(47)}$.

In summary, we found a significant decrease in the risk of NSCL/P after FA fortification in the current multi-ethnic meta-analysis. Although the number of NSCL/P samples was lower than that of total OFC, the absence of both between-study heterogeneity and publication bias, plus the robustness of this result in the sensitivity analysis, leads us to conclude that our finding is evidence of the beneficial effect of FA fortification policies.

\section{Acknowledgements}

Financial support: This research received no specific grant from any funding agency in the public, commercial or notfor-profit sectors. Conflict of interest: All of the authors declare no conflict of interest. Authorship: N.M. and J.S. designed the study, performed the bibliographical search and data extraction. J.S. performed the statistical analyses. N.M., R.P., L.C. and J.S. (i.e. all authors) wrote, revised and approved the final version of the manuscript. Ethics of buman subject participation: Not applicable.

\section{Supplementary material}

To view supplementary material for this article, please visit https://dx.doi.org/10.1017/S1368980017000878

\section{References}

1. Mossey P (2007) Epidemiology underpinning research in the aetiology of orofacial clefts. Orthod Craniofac Res 10, 114-120.

2. IPDTOC Working Group (2011) Prevalence at birth of cleft lip with or without cleft palate: data from the International Perinatal Database of Typical Oral Clefts (IPDTOC). Cleft Palate Craniofac J 48, 66-81.

3. Watkins SE, Meyer RE, Strauss RP et al. (2014) Classification, epidemiology and genetics of orofacial clefts. Clin Plastic Surg 41, 149-163.

4. Schutte BC \& Murray JC (1999) The many faces and factors of orofacial clefts. Hum Mol Genet 8, 1853-1859.

5. Dixon MJ, Marazita ML, Beaty TH et al. (2011) Cleft lip and palate: understanding genetic and environmental influences. Nat Rev Genet 12, 167-178.

6. Vieira AR (2008) Unraveling human cleft lip and palate research. J Dent Res 87, 119-125.

7. Bhaskar LV, Murthy J \& Venkatesh Babu G (2011) Polymorphisms in genes involved in folate metabolism and orofacial clefts. Arch Oral Biol 56, 723-737.
8. Lucock M (2000) Folic acid: nutritional biochemistry, molecular biology, and role in disease processes. Mol Genet Metab 71, 121-138.

9. Jones PA \& Takai D (2001) The role of DNA methylation in mammalian epigenetics. Science 293, 1068-1070.

10. Bogdanović O \& Veenstra GJ (2009) DNA methylation and methyl-CpG binding proteins: developmental requirements and function. Chromosoma 118, 549-565.

11. Figueiredo RF, Figueiredo N, Feguri A et al. (2015) The role of the folic acid to the prevention of orofacial cleft: an epidemiological study. Oral Dis 21, 240-247.

12. Badovinac RL, Werler MM, Williams PL et al. (2007) Folic acid-containing supplement consumption during pregnancy and risk for oral clefts: a meta-analysis. Birth Defects Res $A$ Clin Mol Teratol 79, 8-15.

13. Butali A, Little J, Chevrier C et al. (2013) Folic acid supplementation use and the MTHFR C677T polymorphism in orofacial clefts etiology: an individual participant data pooled-analysis. Birth Defects Res A Clin Mol Teratol 97, 509-514.

14. Molina-Solana R, Yáñez-Vico RM, Iglesias-Linares A et al. (2013) Current concepts on the effect of environmental factors on cleft lip and palate. Int J Oral Maxillofac Surg $\mathbf{4 2}$, $177-184$

15. De-Regil LM, Peña-Rosas JP, Fernández-Gaxiola AC et al. (2015) Effects and safety of periconceptional oral folate supplementation for preventing birth defects. Cochrane Database Syst Rev 12, CD007950.

16. Cortes F, Mellado C, Pardo RA et al. (2012) Wheat flour fortification with folic acid: changes in neural tube defects rates in Chile. Am J Med Genet A 158A, 1885-1890.

17. López-Camelo JS, Orioli IM, da Graça Dutra M et al. (2005) Reduction of birth prevalence rates of neural tube defects after folic acid fortification in Chile. Am J Med Genet A 135A, 120-125.

18. López-Camelo JS, Castilla EE, Orioli IM et al. (2010) Folic acid flour fortification: impact on the frequencies of 52 congenital anomaly types in three South American countries. Am J Med Genet A 152A, 2444-2458.

19. Castilla EE, Orioli IM, Lopez-Camelo JS et al. (2003) Preliminary data on changes in neural tube defect prevalence rates after folic acid fortification in South America. Am J Med Genet A 123A, 123-128.

20. Nazer HJ, Cifuentes OL, Aguila RA et al. (2007) Effects of folic acid fortification in the rates of malformations at birth in Chile. Rev Med Chil 135, 198-204.

21. Nazer HJ \& Cifuentes OL (2014) Prevalence of congenital malformations at birth in Chilean maternity hospitals. Rev Med Chil 142, 1150-1156.

22. Golalipour MJ, Vakili MA \& Kaviani N (2014) Reduction in non syndromic oral clefts following mandatory flour fortification with folic acid in Northern Iran. Med J Islam Repub Iran 28, 29.

23. Wells GA, Shea B, O'Connell D et al.2014) The NewcastleOttawa Scale (NOS) for assessing the quality of nonrandomised studies in meta-analyses. http://www.ohri. $\mathrm{ca} /$ programs/clinical_epidemiology/oxford.asp (accessed August 2016).

24. Huedo-Medina TB, Sánchez-Meca J, Marín-Martínez F et al. (2006) Assessing heterogeneity in meta-analysis: $Q$ statistic or $I^{2}$ index? Psychol Methods 11, 193-206.

25. Borenstein M, Hedges LV, Higgins JPT et al. (2009) Introduction to Meta-Analysis. Chichester: John Wiley \& Sons Ltd.

26. Food Fortification Initiative (2016) Mandatory Cereal Grain Legislation. http://www.ffinetwork.org/global_progress/index. php (accessed August 2016).

27. Coburn KM \& Vevea JL (2015) Publication bias as a function of study characteristics. Psychol Methods 20, 310-330. 
28. Wallace BC, Dahabreh IJ, Trikalinos TA et al. (2012) Closing the gap between methodologists and end-users: $\mathrm{R}$ as a computational back-end. J Stat Softw 49, 1-15.

29. Ray JG, Meier C, Vermeulen MJ et al. (2003) Association between folic acid food fortification and congenital orofacial clefts. J Pediatr 143, 805-807.

30. Simmons CJ, Mosley BS, Fulton-Bond CA et al. (2004) Birth defects in Arkansas: is folic acid fortification making a difference? Birth Defects Res A Clin Mol Teratol 70, 559-564.

31. Canfield MA, Collins JS, Botto LD et al. (2005) Changes in the birth prevalence of selected birth defects after grain fortification with folic acid in the United States: findings from a multi-state population-based study. Birth Defects Res A Clin Mol Teratol 73, 679-689.

32. Hashmi SS, Waller DK, Langlois P et al. (2005) Prevalence of nonsyndromic oral clefts in Texas: 1995-1999. Am J Med Genet A 134A, 368-372.

33. Botto LD, Lisi A, Bower C, Canfield MA et al. (2006) Trends of selected malformations in relation to folic acid recommendations and fortification: an international assessment. Birth Defects Res A Clin Mol Teratol 76, 693-705.

34. Yazdy MM, Honein MA \& Xing J (2007) Reduction in orofacial clefts following folic acid fortification of the US grain supply. Birth Defects Res A Clin Mol Teratol 79, 16-23.

35. Godwin KA, Sibbald B, Bedard T et al. (2008) Changes in frequencies of select congenital anomalies since the onset of folic acid fortification in a Canadian birth defect registry. Can J Public Health 99, 271-275.

36. Sayed AR, Bourne D, Pattinson R et al. (2008) Decline in the prevalence of neural tube defects following folic acid fortification and its cost-benefit in South Africa. Birth Defects Res A Clin Mol Teratol 82, 211-216.
37. Souza J \& Raskin S (2013) Clinical and epidemiological study of orofacial clefts. J Pediatr (Rio J) 89, 137-144.

38. Yang W, Carmichael SL \& Shaw GM (2016) Folic acid fortification and prevalences of neural tube defects, orofacial clefts, and gastroschisis in California, 1989 to 2010. Birth Defects Res A Clin Mol Teratol 106, 1032-1041.

39. Tolarova M (1982) Periconceptional supplementation with vitamins and folic acid to prevent recurrence of cleft lip. Lancet 2, 217.

40. Czeizel AE, Tímár L \& Sárközi A (1999) Dose-dependent effect of folic acid on the prevention of orofacial clefts. Pediatrics 104, e66.

41. Higgins JP (2008) Heterogeneity in meta-analysis should be expected and appropriately quantified. Int J Epidemiol 37, $1158-1160$.

42. Matthews RG, Sheppard C \& Goulding C (1998) Methylenetetrahydrofolate reductase and methionine synthase: biochemistry and molecular biology. Eur J Pediatr 157, Suppl. 2, S54-S59.

43. National Center for Biotechnology Information, National Institutes of Health (2016) dbSNP Short Genetic Variations. http://www.ncbi.nlm.nih.gov/SNP/ (accessed August 2016).

44. Borja-Aburto VH (2000) Estudios ecológicos. Salud Publica Mex 42, 533-538.

45. Leslie EJ \& Marazita ML (2013) Genetics of cleft lip and cleft palate. Am J Med Genet C Semin Med Genet 163C, 246-258.

46. Carinci F, Pezzetti F, Scapoli L et al. (2003) Recent developments in orofacial cleft genetics. J Craniofac Surg 14, 130-143.

47. Johnson CY \& Little J (2008) Folate intake, markers of folate status and oral clefts: is the evidence converging? Int J Epidemiol 37, 1041-1058. 